\title{
A Method of Feature Extraction and Compression of 3D Model
}

\author{
Zuojun $\mathrm{Liu}^{1, \mathrm{a}^{*}}$, Lihong $\mathrm{Li}^{2, \mathrm{~b}} \mathrm{Mi} \mathrm{Yu}^{3, \mathrm{c}}$ \\ ${ }^{1}$ Faculty of Computer and Software Engineering, Huaiyin Institute of Technology, Huai'an, Jiangsu, \\ China \\ ${ }^{2}$ Faculty of Foreign Language, Huaiyin Institute of Technology, Huai'an, Jiangsu, China \\ ${ }^{3}$ Faculty of Foreign Language, Huaiyin Institute of Technology, Huai'an, Jiangsu, China

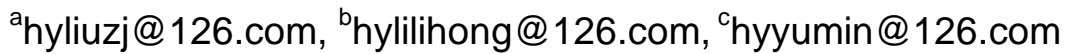

Keywords: Pre-processing; Perspective projection; Fourier transform; Compression

Abstract. Feature extraction is a major concern in 3D retrieval, which decides its performance and efficiency. Therefore, feature extraction and compression become the keys to guarantee the retrieval's performance and speed. The process is as follows: firstly, transform the standard 3D model to 2D images by perspective projection; secondly, transform these images to frequency domain by Fourier Transformation to get the vectors feature; lastly, compress the vectors to six dimensions. This algorithm solves the contradictions between large information and low speed, low information and low performance. Proved by the experiment: the solution is more effective and more rapidly.

\section{Introduction.}

In recent years, with the rapid development internet technology and multimedia technology, all kinds of information is spreading conveniently and rapidly. Face with the huge and complicated information, people hope to be able to find the resources quickly and accurately. Therefore, the technique how to find the model you needed quickly become a research focus.

For the retrieval system of 3D model, feature extraction is the most major and critical technique. So, there are two basic requirements for feature extraction: one is that the feature extracted should be enough to differentiate the various models to guarantee the retrieval efficiency; the other is that the amount of information cannot be too much to guarantee the retrieval speed. Efficiency and speed are constraint reciprocally, which is difficult to meet at the same time.

Therefore, this paper gives a method of feature extraction and compression on 3D model. This method can improve the retrieval efficiency and speed at the same time.

\section{Pretreatment.}

The information, such as location, size and orientation contained in 3D model is uncertain. When we are retrieving, the coordinate system of the example model is more likely different from those in the model database. If we do nothing, the same models can be treated as the different ones, which cause lower retrieval efficiency. So, pretreatment is necessary.

Thoughts of Pretreatment. To realize the 3D model retrieval, the models must be parameterized. Too much variable parameters can cause too much problems, such as increased difficulty of analysis, complicacy and increased computation. These variables are related. We can select much less variables to express 3D model according to the relations of these variables. These much less variables must contain the most information of 3D model. In this paper, a method named principal component analysis which can replace much more variables with much less variables is introduced.

Pretreatment. Assuming a linear equations consists of $p$ dependent variables $y_{1}, y_{2}, \mathrm{~K}, y_{p}$; each equation consists of $m$ independent variables; $m(m<p)$, is the number of independent variables; $x_{i}$ is standard variable whose mean value is 0 and its standard deviation is 1 . The equations is shown as formula (1), 


$$
\left\{\begin{array}{c}
y_{1}=\alpha_{11} x_{1}+\alpha_{12} x_{2}+\alpha_{13} x_{3}+\cdots+\alpha_{1 m} x_{m}+\varepsilon 1 \\
y_{2}=\alpha_{21} x_{1}+\alpha_{22} x_{2}+\alpha_{23} x_{3}+\cdots+\alpha_{2 m} x_{m}+\varepsilon 2 \\
y_{3}=\alpha_{31} x_{1}+\alpha_{32} x_{2}+\alpha_{33} x_{3}+\cdots+\alpha_{3 m} x_{m}+\varepsilon 3 \\
\quad \cdots \cdots \\
y_{p}=\alpha_{p 1} x_{1}+\alpha_{p_{2}} x_{2}+\alpha_{p 3} x_{3}+\cdots+\alpha_{p m} x_{m}+\varepsilon_{p}
\end{array}\right.
$$

The equations can also be expressed as a matrix form. The equation is shown as formula (2).

$$
Y=A X+e
$$

In this formula, $X$ is a dependent variables; $A$ is a load matrix; $\alpha_{i j}$ is the factor loading. The equation of the covariance matrix is shown as formula (3),

$$
S=A A^{\prime}+D
$$

In the formula, $D$ is the covariance matrix of $e$. The covariance matrix is just the correlation coefficient matrix because that $X$ is standardized. The equation is shown as formula (4),

$$
R=A A^{\prime}+D
$$

The greater of the absolute value of $\alpha_{i j}$ is, the stronger of the relation between the combination vector $z_{i}$ and the original vector $x_{i}$ is. Through a method of coordinate transformation, the original $p$ relevant variables $y_{i}$ is converted into unrelated ones. The equation is shown as formula (5),

$$
\left\{\begin{array}{c}
z_{1}=\beta_{11} y_{1}+\beta_{12} y_{2}+\beta_{13} y_{3}+\cdots+\beta_{1 p} y p \\
z_{2}=\beta_{21} y_{1}+\beta_{22} y_{2}+\beta_{23} y_{3}+\cdots+\beta_{2 p} y_{p} \\
z_{3}=\beta_{31} x_{1}+\beta_{32} x_{2}+\beta_{33} x_{3}+\cdots+\beta_{3 p} x_{p} \\
\quad \cdots \cdots \\
z_{p}=\beta_{p 1} y_{1}+\beta_{p_{2}} y_{2}+\beta_{p 3} y_{3}+\cdots+\beta_{p p} y_{p}
\end{array}\right.
$$$$
\text { ) }
$$

The variance of $z_{1}$ is the largest among all of the linear combinations of $y_{1}, y_{2}, y_{3}, \mathrm{~K}, y_{p}$. So, $z_{1}$ is the first principal component. Likewise the variance of $z_{2}, z_{3}, \mathrm{~K}, z_{p}$ follow, which respectively as the second and the third principal component. Setting $R=\widetilde{A} \widetilde{A}^{\prime}$, we can get $p$ eigenvalues of $R$ and corresponding eigenvectors $\beta_{k}=\left(\beta_{k 1}, \beta_{k 2}, \beta_{k 3}, \mathrm{~K}, \beta_{k p}\right)^{\prime}, k=1,2,3, \mathrm{~K}, p$. So, we can get formula (6).

$$
R=\left(\beta_{1}, \beta_{2}, \beta_{3}, \mathrm{~K}, \beta_{k}\right)\left[\begin{array}{l}
\beta_{1}{ }^{\prime} \\
\beta_{2}{ }^{\prime} \\
\beta_{3}{ }^{\prime} \\
\mathrm{M} \\
\beta_{p^{\prime}}{ }^{\prime}
\end{array}\right]=\widetilde{A} \widetilde{A}^{\prime}
$$

The solution of load matrix can be calculated through formula (6). The calculation equation is shown as formula (7),

$$
\widetilde{A}=\left[\begin{array}{cc}
\alpha_{11} \alpha_{12} \mathrm{~L} & \alpha_{1 p} \\
\alpha_{21} \alpha_{22} \mathrm{~L} & \alpha_{2 p} \\
\mathrm{~L} \mathrm{~L} & \\
\alpha_{p 1} \alpha_{p 2} \mathrm{~L} & \alpha_{p p}
\end{array}\right]=\left[\begin{array}{cccc}
\beta_{11} \sqrt{\lambda_{1}} & \beta_{12} \sqrt{\lambda_{2}} & \mathrm{~L} & \beta_{1 p} \sqrt{\lambda_{p}} \\
\beta_{21} \sqrt{\lambda_{1}} & \beta_{22} \sqrt{\lambda_{2}} & \mathrm{~L} & \beta_{2 p} \sqrt{\lambda_{p}} \\
\mathrm{~L} \mathrm{~L} & & & \\
\beta_{p 1} \sqrt{\lambda_{1}} & \beta_{p 2} \sqrt{\lambda_{2}} & \mathrm{~L} & \beta_{p p} \sqrt{\lambda_{p}}
\end{array}\right]
$$

When calculating the load matrix, we only choose the first $m$ eigenvalues and the corresponding eigenvectors. So, we can get a load matrix with $m$ public variable, as shown in formula (8),

$$
\widetilde{A}=\left[\begin{array}{cc}
\alpha_{11} \alpha_{12} \mathrm{~L} & \alpha_{1 m} \\
\alpha_{21} \alpha_{22} \mathrm{~L} & \alpha_{2 m} \\
\mathrm{LL} & \\
\alpha_{p 1} \alpha_{p 2} \mathrm{~L} & \alpha_{p m}
\end{array}\right]=\left[\begin{array}{cccc}
\beta_{11} \sqrt{\lambda_{1}} & \beta_{12} \sqrt{\lambda_{2}} & \mathrm{~L} & \beta_{1 m} \sqrt{\lambda_{m}} \\
\beta_{21} \sqrt{\lambda_{1}} & \beta_{22} \sqrt{\lambda_{2}} & \mathrm{~L} & \beta_{2 m} \sqrt{\lambda_{m}} \\
\mathrm{LL} & & & \\
\beta_{p 1} \sqrt{\lambda_{1}} & \beta_{p 2} \sqrt{\lambda_{2}} & \mathrm{~L} & \beta_{p m} \sqrt{\lambda_{m}}
\end{array}\right]
$$

After the analysis of the principal component, the vertex of $3 \mathrm{D}$ model with the most information is distributed on the first spindle as the first principal component etc. So, the main information of the 3D model is respectively distributed on three axes $X, Y, Z$. 


\section{Feature Extraction.}

Feature extraction is the most important and critical part in 3D model retrieval, whose efficiency is directly related to the whole retrieval system.

Perspective Projection. After pretreatment, the effect of pretreatment is shown in figure 1. a.

In order to obtain the features, the 3D model is projected from several directions. A tetrahedron is used to surround the 3D model after pretreatment, such as figure 1. b. Six points $K 、 L 、 M 、 K^{\prime} 、 L^{\prime}$ 、 $M^{\prime}$ can be got when the three coordinate axes run across the tetrahedron. The six points can be used as the viewpoint. By projecting, we can get six images of 3D model from six viewpoints. The 3D model is transformed from 3D space into six images in 2D space with size of $\mathrm{M}=\mathrm{N}=64$. The attribute $\mathrm{f}(\mathrm{a}, \mathrm{b})$ of each point $(a, b)$ of the image is the closest distance between the point on the direction perpendicular to the plane and the viewpoint, as shown in Eq. (9),

$$
f(a, b)=\sqrt{A^{2}+B^{2}}
$$

In formula (9), $A$ is the shortest vertical distance between point $(a, b)$ and the surface of the $3 D$ model. $\mathrm{B}$ is the distance between point $(\mathrm{a}, \mathrm{b})$ and the center $\mathrm{r}_{Y}$ of the image.

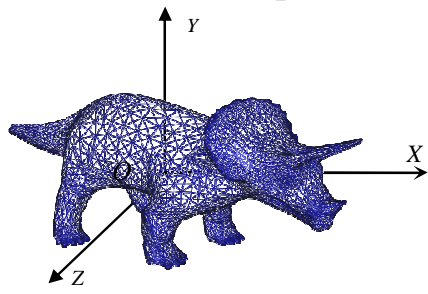

a

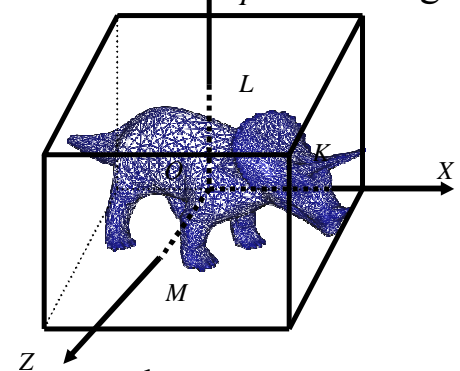

b

Fial perspective proiection transformation

Feature Extraction. After projection, six images $(M \times N)$ can be got. At the same time, the 3D model is transformed from 3D space into 2D space. Now, these images will continue to be transformed from airspace into frequency domain in order to get the features of the 3D model by DFT. The time complexity of direct FT is $O\left(M^{2} N^{2}\right)$. The equation is shown as formula (10),

$$
F(p, q)=\frac{1}{M} \sum_{x=0}^{M-1}\left(\frac{1}{N} \sum f(x, y) e^{-j 2 \pi p y / N}\right) e^{-j 2 \pi p x / M}
$$

The time complexity of two times 1D FT is $O(M \times N \times(M+N))$, which is more lower than the direct Fourier transform. After Fourier transform, the six images of 3D model is transformed into energy distribution chart in frequency domain. One percent eight of the low-frequency energy area can be extracted to express the 3D model. The dimension of eigenvector of the image is $6 \times(64 / 8) \times(64 / 8)=384$, as shown as formula $(11)$,

$$
\begin{aligned}
& \left(v_{00}^{1}, v_{00}^{2}, \mathrm{~L} v_{00}^{6}, v_{01}^{1}, v_{01}^{2}, \mathrm{~L} v_{01}^{6}, \mathrm{~L}, v_{07}^{1}, v_{07}^{2}, \mathrm{~L} v_{07}^{6},\right. \\
& v_{10}^{1}, v_{10}^{2}, \mathrm{~L} v_{10}^{6}, v_{11}^{1}, v_{11}^{2}, \mathrm{~L} v_{11}^{6}, \mathrm{~L}, v_{17}^{1}, v_{17}^{2}, \mathrm{~L} v_{17}^{6}, \\
& \mathrm{~L}, \mathrm{~L}, \mathrm{~L}, \mathrm{~L}, \mathrm{~L}, \mathrm{~L}, \mathrm{~L}, \mathrm{~L}, \mathrm{~L}, \mathrm{~L}, \mathrm{~L}, \mathrm{~L}, \mathrm{~L}, \mathrm{~L}, \\
& \left.v_{70}^{1}, v_{70}^{2}, \mathrm{~L} v_{70}^{6}, v_{71}^{1}, v_{71}^{2}, \mathrm{~L} v_{71}^{6}, \mathrm{~L}, v_{77}^{1}, v_{77}^{2}, \mathrm{~L} v_{77}^{6},\right)
\end{aligned}
$$

In the formula, $v_{x y}^{i}$ is the energy value of image $i$ when the frequency variable is $x, y$.

Vector Compression. A problem appeared that the dimension of eigenvector is much larger. The dimension of the eigenvector must be reduced. The eigenvector of each image can be compressed into a 1D. The 1D eigenvector must ensure the greatest degree of differentiation. Fisher discriminant criterion can be used to find a line with best degree of differentiation. The high dimension vector can be projected onto the line to be transformed into 1 dimension.

A sample set containing $N$ sample vector $x_{1}, x_{2}, \mathrm{~K}, x_{N}$ can be selected. The sample vector in the eigenvector set can be divided into three categories. $N_{1}$ is the first subset constituting with satisfied 
samples. $N_{2}$ is the second subset constituting with unsatisfied samples. A scalar $y_{n}$ can be got to do a linear combination with the component of $x_{n}$. The equation is shown as formula (12),

$$
y_{n}=w^{T} x_{n} \quad, n=1,2, \mathrm{~K}, N_{i}
$$

So we can get a 1D sample set with $N$ samples. The set can be divided into two subset $X_{1}$ and $X_{2}$. If $\|w\|=1, y_{n}$ is just the projection of $x_{n}$ onto a line $w$. The direction of line $w$ is very important, which decided the degree of separation of sample projection. That's to say, the direction can directly decide the recognition effect. The question is how to find a line with best projection direction. In mathematics, the question is how to find a best transformation vector $w^{*}$.

$w^{*}$ can be got by using Fisher criterion. The equation is shown as formula (13),

$w^{*}=S_{w}^{-1}\left(m_{1}-m_{2}\right)$

In formula (13), $m_{1}$ is a mean vector of satisfied sample. The equation is shown as formula (14),

$$
m_{i}=\frac{1}{N_{i}} \sum_{x \in X_{i}} x, i=1,2
$$

$S_{w}^{-1}$ is the inverse matrix of $S_{w}$, which is a discrete degree matrix in total class. The equation of $S_{w}$ is shown as formula (15),

$$
S_{w}=S_{1}+S_{2}
$$

$S_{i}$ is a discrete degree matrix in sample class, as shown in formula(16),

$$
S_{i}=\sum_{x \in X_{i}}\left(x-m_{i}\right)\left(x-m_{i}\right)^{T}, i=1,2
$$

$w^{*}$ is the solution when Fisher criterion function gets the great value, and also the best project direction from high dimension to one dimension. Given the projection direction, the high dimension sample can be mapped into one dimension vector.

The eigenvector of each image maps into a one dimensional vector. Six images of each 3D model combine into a six dimension vector $\left(v_{0}, v_{1}, v_{2}, v_{3}, v_{4}, v_{5}\right)$. All of the most important information of a 3D model is reserved. At the same time, the dimension of eigenvector is compressed.

\section{Experimental Verification.}

In order to test the efficiency and performance of above algorithm, the eigenvector is inputted into a network classifier to realize 3D model retrieval. Three kinds of 3D models are selected from the model lib: table models, rabbit models and lamp models. The number of these models is very larger, and the models are similar.

Experimental Results. Here, the experimental result of tables is provided. The others are similar to this. Table 1 gives the experimental results. The result is sorted according to the similarity. It can be seen from table 1 that the algorithm achieves perfect performance.

Table1 Performance Test

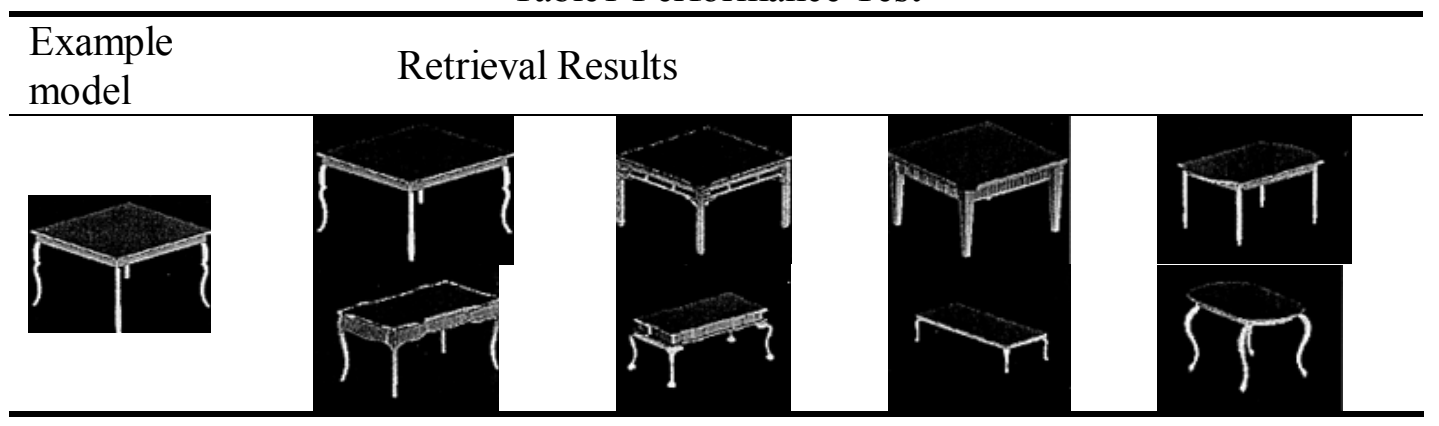


Table 2 provides four times: pretreatment time, feature extraction and compression time, retrieval time, and the total times.

Table 2 Time Test

\begin{tabular}{llll}
\hline Pretreatment $(\mathrm{s})$ & Pretreatment $(\mathrm{s})$ & Retrieval Time $(\mathrm{s})$ & Total Times $(\mathrm{s})$ \\
\hline 0.007 & 0.010 & 0.009 & 0.026 \\
\hline
\end{tabular}

Experimental Analysis. Seen from table 1 and table 2, this method introduced in this paper works with higher retrieval performance and lower time complexity. It can solve the contradictions that the retrieval performance and retrieval speed can't get at the same time.

\section{Conclusions.}

This paper discussed the method of feature extraction and compression of $3 \mathrm{D}$ model. High dimension vector is extracted firstly. Then, the high dimension vector is compressed. This algorithm realizes the retrieval of 3D model with high speed and perfect performance. More important, the algorithm solves the contradiction of performance and efficiency.

\section{Reference}

[1]Kearns M, Valiant L G.Learning Boolean Formulae or Factoring[R].Technical Report TR-1488, Harvard University Aiken Computation Laboratory, 2012,43(3):28-33

[2]Raj Dharmarajan Jyer Jr.An Eifficient Boosting Algorithm for Combining Preferences[D]. Massachusetts Institute of Technology, 2007,41(7):28-33

[3]Alexander Goltsev, Donald C.wunsch.Generalization of Features in the Assembly Neural Networks [J].International Journal of Neural Systems (IJNS), 2013,24(4):39-56

[4]I.Guyon.Applications of Neural Networks of Character Recognition [J]. International Journal of Pattern Recognition and Artificial Intelligence (IJPRAI), 2013,44(2):353-382

[5]Liu C L , Kazuki N, Hiroshi S. Handwritten Digit Recognition: Investigation of Normalization and Feature Extraction Techniques[J].Pattern Recognition, 2010,56(2):265-279 